\title{
ORDERED CAUCHY SPACES
}

\author{
D. C. KENT and R. VAINIO \\ Department of Pure and Applied Mathematics \\ Washington State University \\ Pullman, WA 99164-2930
}

(Received May 1, 1985)

ABSTRACT: This paper is concerned with the notion of "ordered Cauchy space" which is given a simple internal characterization in Section 2. It gives a discription of the category of ordered Cauchy spaces which have ordered completions, and a construction of the "fine completion functor" on this category. Sections 4 through 6 deals with certain classes of ordered Cauchy spaces which have ordered completions; examples are given which show that the fine completion does not preserve such properties as uniformizability, regularity, or total boundedness. From these results, it is evident that a further study of ordered Cauchy completions is needed. KEY WORDS AND PHRASES: Ordered Cauchy spaces, ordered completions, uniformizability, regularity, total boundedness.

1980 AMS MATHEMATICS CLASSIFICATIONS CODES: 54A20, 54ґ15.

\section{0 . INTRODUCTION.}

Cauchy spaces provide a completion theory much more general than that obtained from metric or uniform spaces, and much simpler than that arising from uniform convergence spaces. R. Ball [1], [2] has shown the applicability of Cauchy spaces in the study of lattice completions; the compatibility between lattice and Cauchy structures is obtained by requiring the lattice operations to be Cauchy-continuous. While this compatibility criterion is quite natural it is not appropriate for more general order structures, such as partial orders, and may be too restrictive even for some lattice applications.

L. Nachbin [9] has defined an "ordered uniform space" by requiring that the uniform and order structures arise from a common source, that being a quasi-uniform structure on the same underlying set. We adapt Nachbin's method to define an "ordered uniform convergence space", and from this the defintion of "ordered Cauchy structure" follows naturally. The resulting notion of "ordered Cauchy space" is given a simple internal characterization in Section 2.

The development of a completion theory for ordered Cauchy spaces is complicated by the fact that some ordered Cauchy spaces do not have ordered completions. In Section 3, we describe the category of ordered Cauchy spaces which have ordered completions, and construct the "fine completion functor" on this category. The remaining sections examine certain classes of ordered Cauchy spaces which have ordered completions; examples are given which show that the fine completion does not preserve such properties as uniformizability, regularity, or total boundedness. From these results, it 1s evident that a further study of ordered Cauchy completions is needed. 


\section{PRELIMINARIES.}

Let $X$ be a set, and $F(X)$ the set of all (proper) filters on $X$. If F, $\& \in F(X)$, and $F \cap G \neq \phi$ for all $F \in F, G \in \&$, then $U \&$ denotes the filter on $X$ generated by $\{F \cap G: F \in \mathcal{F}, G \in \&\}$. On the other hand, if $F \cap G=\phi$ for some $F \in \mathcal{F}$ and some $G \in \mathcal{Z}$, we say that $\mathcal{F} \&$ fafls to exist. If $A \subseteq X, A \neq \phi$, we denote by $\langle A>$ the filter of all oversets of $A$; one departure from this convention are the fixed ultrafilters, which are denoted by $\dot{x}$, for $x \in \mathbf{x}$.

Turning to the product set $\mathrm{X} x \mathrm{x}$, the diagonal $\{(\mathrm{x}, \mathrm{x}): \mathbf{x} \in \mathrm{X}\}$ is designated by $\Delta$. Compositions are defined as follows: for subsets $A, B$ of $X \times X$, $A \circ B=\{(x, y):$ there is $z \in X$ such that $(x, z) \in A$ and $(z, y) \in B\}$. Compositions and inverses of filters on $X \times X$ are defined in the obvious way. If $A \subseteq X \times X$ and $H \subseteq X$, then $A(H)=\{y \in H:(x, y) \in A$ for some $x \in H\}$; if, in addition, $G \in F(X \times X)$ and $\& \in F(X)$, then $A(\&), G(H)$, and $G(A)$ denote the filters on $X$ generated by the sets $\{A(H): H \in F\},\{A(H): A \in G\}$, and $\{A(H): A \in \mathcal{F}, H \in Z\}$, respectively.

DEFINITION 1.1. A set $\sigma$ of filters on $X \times x$ is called a quasi-uniform convergence structure on $\mathrm{X}$ if:

$$
\begin{aligned}
& \left(u_{1}\right) \dot{\Delta} \in \sigma \\
& \left(u_{2}\right) \quad z \cap \& \in \sigma \text { whenever } \exists, \& \in \sigma \\
& \left(u_{3}\right) \xi \circ \& \in \sigma \text { whenever } \exists, \& \in \sigma \text { and } \xi \circ \& \text { exists } \\
& \left(u_{4}\right) \quad z \in \sigma \text { and } z \subseteq \& \text { implies } \& \in \sigma .
\end{aligned}
$$

If $\sigma$ satisfies the additional condition

$$
\left(u_{5}\right) ; \in \sigma \text { implies } z^{-1} \in \sigma
$$

then $\sigma$ is called a uniform convergence structure on $\mathrm{X}$.

Quasi-uniform convergence structures are natural generalizations of quasiuniformities. Uniform convergence structures were introduced by Cook and Fischer [3]; another term of ten used for the same concept is "pseudouniforme Struktur" (see [6]).

A subset $\sigma^{\prime}$ of a quas1-uniform convergence structure $\sigma$ is called a base for $\sigma$ if, for each $\& \in \sigma$, there is $B \in \sigma^{\prime}$ such that $\mathscr{B} \subseteq \&$. One convenient rise for any quasi-uniform convergence structure $\sigma$ is $\sigma_{\Delta}=\{\& \in \sigma: \& \subseteq<\Delta>\}$.

The set $Q_{u}(X)$ of all quasi-uniform convergence structures on $X$ forms? complete lattlce under the natural ordering (the dual of set inclusion). If $\rho, \sigma \in Q_{u}(X)$, then $\rho \wedge \sigma$ has for a base all finite compositions $\&_{1} \circ \ldots \circ \&_{n}$. where for each $j, \&_{j} \in \rho_{\Delta}$ or $\&_{j} \in \sigma_{\Delta^{*}}$. If $\sigma \in Q_{u}(X)$, then $\sigma v \sigma^{-1}$ is the uniform convergence structure on $\mathrm{Y}$ induced by $\sigma$. The lattice operations " $V$ " an!

$" \wedge "$, applied to quasi-uniform convergence structures (including uniform convergence structures) will always be taken relative to the lattice $Q_{u}(X)$.

DEFINITION 1.2. A set $C$ of filters on $X$ is a Cauchy structure on $X$ if : $\left(c_{1}\right) \dot{x} \in C$, for all $x \in \mathrm{x}$ 


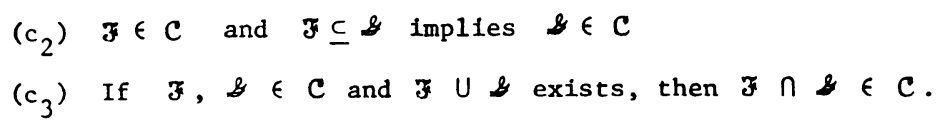

A subset $B$ of a Cauchy structure $C$ is a base for $C$ if, for each $\epsilon$,

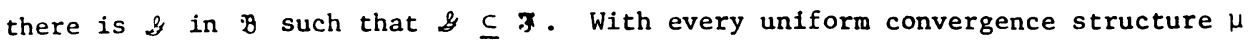
on $X$, there is associated the induced Cauchy structure $C_{\mu}=\{F \in F(X)$ : $3 x \in \mu\}$. On the other hand, one can associate with each Cauchy structure a finest compatible uniform convergence $\mu^{C}$, with base consisting of all finite intersections of filters of the form $(\exists \times) \cap \dot{\Delta}$, where $\in C$ (one can show that this set of finite intersections is closed under finite compositions). Also associated with a Cauchy structure $C$ on $X$ is the convergence structure ${ }^{q_{C}}$, defined by: $\rightarrow x$ in $\left(x, q_{C}\right)$ iff $\cap \dot{x} \in C$.

If $f:\left(X_{1}, C_{1}\right) \rightarrow\left(X_{2}, C_{2}\right)$ is a function from one Cauchy space to another, then $f$ is Cauchy-continuous if $f(F) \in C_{2}$ whenever $\nleftarrow \in C_{1}$.

We next consider the notation and terminology associated with an order relation $\leq$ on $X$. We shall always assume that $(X, \leq)$ is a poset, and we identify the order relation with its graph $\theta=\{(\mathrm{x}, \mathrm{y}): \mathrm{x} \leq \mathrm{y}\} \subseteq \mathrm{x} \mathrm{x} \mathrm{x}$. Indeed, it will usually be convenient to designate the poset by $(X, \theta)$ rather than the more conventional notation $(\mathrm{X}, \leq)$.

Let $(X, \theta)$ be a poset; let $x \in X, A \subseteq X$, and $F \in F(X)$. Then $\theta(x)=\theta(\{x\}), \theta(A)$, and $\theta(\xi)$ are the increasing hulls of $x, A$, and $\bar{F}$, respectively. The decreasing hulls are $\theta^{-1}(\mathrm{x}), \theta^{-1}(\mathrm{~F})$, respectively. The convex hul1 of $A$ is $A^{\wedge}=\theta(A) \cap Q^{-1}(A)$, and $A$ is convex if $A=A^{\wedge}$; similar notation applies to filters. A Cauchy space $(X, C)$ is locally convex if ${\Im^{\wedge}}^{\wedge} C$ whenever $\bar{Z} \in \mathrm{C}$.

If $\left(x_{1}, \theta_{1}\right)$ and $\left(x_{2}, \theta_{2}\right)$ are posets, a function $f:\left(x_{1}, \theta_{1}\right) \rightarrow\left(x_{2}, \theta_{2}\right)$ is increasing if $f\left(\theta_{1}\right) \subseteq \theta_{2}$ (equivalently, if $f\left(x_{1}\right) \leq f\left(x_{2}\right)$ whenever $\left.x_{1} \leq x_{2}\right)$.

\section{ORDERED CAUCHY SPACES.}

Throughout this section, $(\mathrm{X}, \theta)$ will be àn arbitrary poset.

DEFINITION 2.1. Let $(X, \mu)$ be a uniform convergence space. Then $(X, \theta, \mu)$ is an ordered uniform convergence space if there is a quasi-uniform convergence structure $\sigma$ on $x$ such that $\langle\theta\rangle \in \sigma, \mu=\sigma \vee \sigma^{-1}$, and $\theta=U\{\cap G: G \in \sigma\}$.

DEFINITION 2.2. Let $(X, C)$ be a Cauchy space. Then $(X, \theta, C)$ is an ordered Cauchy space (abbreviated o.c.s.) If there is a uniform convergence structure $\mu$ on $X$ such that $(X, \theta, \mu)$ is an ordered uniform convergence space, and $C=C_{\mu}$.

We see that $(X, \theta, C)$ is an o.c.s. iff $C$ and $\theta$ are both determined by some quasi-uniform convergence structure $\sigma$ on $X$ (in the sense described in the preceding definitions). We shall obtain a more precise characterization of of this concept by making use of a particular quasi-uniform convergence structure $\sigma \theta, \mathrm{C}$ constructed directly from $\theta$ and $C_{\theta}$. Let $\sigma_{\theta}=\{\& \in \mathrm{F}(\mathrm{X} \times \mathrm{X}):\langle\theta\rangle \subseteq \&\}$ be the quasi-uniform convergence structure on $X$ generated by $\theta$. If $C$ is a Cauchy 
structure on $(x, \theta)$, we define $\sigma_{\theta, C}=\sigma_{\theta} \wedge \mu ;$ we also define the associated uniform convergence structure $\mu_{\theta, C}=\left(\sigma_{\theta, C}\right) \vee\left(\sigma_{\theta, C}\right)^{-1}$.

LEMMA 2.3. Let $(\mathrm{X}, \theta)$ be a poset. For arbitrary filters $\&$ on $\mathrm{X}$, the following statements are equivalent:

(a) $\cup(\xi) \cup \&$ exists.

(b) $\xi \cup \theta^{-1}(\&)$ exists.

(c) $\theta($ ( ) $) \cup \theta^{-1}(\&)$ exists.

(d) $(\pi \times \&) \cup<\theta>$ exists.

(e) $(\pi \times \mathfrak{F}) \circ<\theta>0<\& \times \&>$ exists.

Let $(\mathrm{X}, \theta)$ be a poset and $\mathrm{C}$ a Cauchy structure on $\mathrm{X}$. A relation $\leqslant$ is defined on $C$ as follows: If $\exists, \& \in C$, then $\lesssim \&$ iff there is a finite set of filters $H_{1}, \ldots, A_{n}$ in $C$ such that $\left.\theta(\xi) \cup H_{1}, \ldots, \theta_{n}\right) \cup \&$ all exist. This relation is clearly reflexive and transitive, but not generally anti-symmetric.

PROPOSITION 2.4. Let $(X, \theta)$ be a poset, $C$ a Cauchy structure on $X$. Then $\sigma_{\theta, C}$ has a base of filters of the form $\left(\cap\left\{\theta^{-1}\left(j_{j}\right) \times \theta\left(\xi_{j}\right): j=1, \ldots, n\right\}\right) \cap\langle\theta\rangle$, where $z_{j} \leq \mathscr{y}_{j}, 1 \leq j \leq n$.

PROOF: Assume $\leqslant \&$ in $C$. Then there is $\sharp_{1}, \ldots, \sharp_{n}$ in $C$ such that $\theta\left(\right.$ (अ) $\cup H_{1}, \ldots, \theta\left(H_{n}\right) \cup \&$ all exist. Thus $\theta^{-1}(\xi) \times \theta(\&)=$

$$
\langle\theta\rangle \circ[3 \times \mathbf{3}] \circ\langle\theta\rangle\left[\mathrm{H}_{1} \times \mathrm{H}_{1}\right] \circ\langle\theta\rangle \ldots \circ\langle\theta\rangle \circ[\& \times \&] \circ\langle\theta\rangle
$$

exists; since each component filter in this composition is in either $\sigma_{\theta}$ or $\mu^{C}$, $\theta^{-1}(\Im) \times \theta(\xi) \in \sigma \quad, C$. Finite intersections of such filters are also in $\sigma, C$, and so $\sigma Q, C$ contains all filters of the indicated form. Furthermore, $\sigma_{\theta, C}$ has a base of filiers of the form $\langle\theta\rangle \circ a_{1} \circ\langle\theta\rangle \circ \ldots \circ\langle\theta\rangle \circ a_{n} \circ\langle\theta\rangle$, where each $a_{j}$ is a basis filter for $\mu^{C}$, that is, a finite intersection of filters of the form $\bar{F}$, where $Z \in C$. A straightforward set-theoretic argument shows that all such basic filters for $\sigma_{\theta, C}$ can be expressed in the form specified in the statement of the proposition.

PROPOSITION 2.5. Let $(X, \theta)$ be a poset, $C$ a Cauchy structure on $X$, and $z, z \in C$. Then $z \leqslant \&$ iff $z \times \& \in \sigma_{\theta, C}$.

PROOF. If $z<\&$, the preceding proof shows that $z \times \&$ is in $\sigma_{\theta, C}$ since $\theta^{-1}(F) \times \theta(\&) \subseteq F \times \&$. Conversely, suppose $x \& \in \sigma_{\theta, C}$. Then, by Proposition 2.4, there are filters $\pi_{1}, \ldots, \pi_{n}$ and $s_{1}, \ldots, s_{n}$ in $C$ with $\pi_{j} \leq \xi_{j}, 1 \leq j \leq n$, such that $\left(\cap\left\{\theta^{-1}\left(F_{j}\right) \times \theta\left(\&_{j}\right): j=1, \ldots, n\right\}\right) \cap\langle\theta>\subseteq$ F 8 . If $(3 \times \&) \cup\langle\theta\rangle$ exists, then $\leqslant \&$ is clear. Otherwise, for some $j,(\xi \times \&) \cup\left(\theta^{-1}\left(\xi_{j}\right) \times \theta\left(\xi_{j}\right)\right)$ exists. This implies $\exists \leqslant z_{j} \leqslant \xi_{j} \leqslant \&$, and the proof is complete.

PROPOSITION 2.6. Let $(X, \theta)$ be a poset and $C$ a Cauchy structure on $X$. Then $(x, \theta, C)$ is an o.c.s. iff $\theta=U\left\{\cap a: a \in \sigma \theta_{\theta, C}\right\}$ and $\mu, C$ is compatible with $C$.

PROOF. If the two conditions are satisfied, then $(X, \theta, C)$ is an o.c.s. by Definitions 2.1 and 2.2 .

Conversely, assume that $(\mathrm{X}, \theta, \mathrm{C})$ is an o.c.s., and let $\mu$ be as in 
Definition 2.2. Then $\theta=U\left\{\cap a: G \in \sigma_{\theta} \wedge \mu\right\} \geq U\left\{\cap a: G \in \sigma_{\theta}, c\right\} \geq \theta$; thus the first condition holds. The second follows because $\mu \leq \mu_{\theta, C} \leq \mu$, and the fact that $\mu$ and $\mu^{C}$ are both compatible with $C$.

PROPOSITION 2.7. If $(X, \theta)$ is a poset and $C$ a Cauchy structure on $X$, then $=U\left\{\cap r_{l}: G \in \sigma \sigma_{\theta, C}\right\}$ iff $\dot{x} \leqslant \dot{y}$ implies $\mathrm{x} \leq \mathrm{y}$.

PROUF. With the help of Proposition 2.5, we have $\dot{x} \leqslant \dot{y} \Leftrightarrow \dot{x} x \dot{y} \in \sigma \sigma_{\theta, C}$ $(x, y) \in J\left\{\cap\left(i: a \in \sigma_{Q, C}\right\}\right.$; the conclusion follows from these observations.

A poset with Cauchy structure is defined to be $\mathrm{T}_{2}$-ordered if $\dot{\mathrm{x}} \leqslant \dot{\mathrm{y}}$ implies $x \leq y$. This terminology coincides with the definition of "T ${ }_{2}$-ordered" for convergence ordered spaces in [8] if we identify a convergence structure as a complete Cauchy structure. It follows from Proposition 1.2, [8] that if (X, $\theta, C)$ is a $\mathrm{T}_{2}$-ordered poset with Cauchy structure, then $\theta$ is closed in $\mathrm{X} \times \mathrm{X}$.

A poset with Cauchy structure $(X, \theta, C)$ which is locally convex is said to satisfy Condition $(O C)_{1}$. If $\cap \& \in C$ whenever $3 \leqslant \&$ and $\& \leqslant 3$, then $(X, \theta, C)$ is said to satisfy Condition $(O C)_{2}$. Finally, if $(X, \theta, C)$ is $\mathrm{T}_{2}$-ordered, then we say that Condition $(\mathrm{OC})_{3}$ is satisfied.

PROPOSITION 2.8. An ordered Cauchy space satisfies conditions $(O C)_{1},(O C)_{2}$, and $(\mathrm{OC})_{3} \cdot$

PROOF. Let $(\mathrm{X}, \theta, \mathrm{C})$ be an ordered Cauchy space. Condition $(\mathrm{OC})_{3}$ is an immediate consequence of Propositions 2.6 and 2.7. Proposition 2.6 also asserts that $\mu_{Q, C}$ is a compatible with $C$; this fact will be used to establish $(O C)_{1}$ and $(O C)_{2}$. If $\in C$, then $G=\langle\theta\rangle \circ(\xi \times F) \circ\langle\theta\rangle=\theta^{-1}(3) \times \theta(F) \in \sigma_{\theta, C}$. Also $a^{-1}=\langle\theta\rangle^{-1} \circ(\xi \times F) \circ\langle\theta\rangle^{-1}=\theta(अ) \times \theta^{-1}(\xi) \in(\sigma, \theta)^{-1}$. Thus $\mathrm{a} \cup \mathrm{a}^{-1}=F^{\wedge} \times 3^{\wedge} \in \sigma_{\theta, \mathrm{C}} \vee\left(\sigma_{\theta, \mathrm{C}}\right)^{-1}=\mu_{\theta, \mathrm{C}}$, and so $\boldsymbol{F}^{\wedge} \in \mathrm{C}$, and (OC) ${ }_{1}$ is satisfied. Finally, assume $\leqslant \&$ and $\& \leq 3$. Then, by Proposition $2.5,3 \times \&$ and $\& \times \exists$ are in $\sigma_{\theta, C}$. Thus $(F \cap \&) \times(\xi \cap \&) \sigma_{\theta, C}$, and this filter, being symmetric, is also in $\mu_{\theta, C}$. Since $\mu_{\theta, C}$ is compatible with $C$, $3 \cap \& \in C$.

THEOREM 2.9. Let $(\mathrm{X}, \theta, \mathrm{C})$ be a poset with Cauchy structure. Then $(X, \theta, C)$ is an o.c.s. iff Conditions $(O C)_{1},\left(O C_{2}\right)$, and $(O C)_{3}$ are satisfied.

PROOF. In view of Propositions $2.6,2.7$, and 2.8 , it remains only to show that $\mathbf{C}$ is compatible with $\mu_{O, C}$ when the three conditions are satisfied. In other words, we must show that $\sharp \mathbf{x} \sharp \in \sigma_{\theta, C}$ implies $\sharp \in C$. We prove this implication first under the assumption that $\&$ is a free filter; later, this restriction will be removed.

If $\sharp x \forall \in \sigma_{\theta, C}$, then by Proposition 2.5 there are filters $\mathbf{z}_{1}, \ldots, \boldsymbol{J}_{\mathrm{n}}$ and $\&_{1}, \ldots, \&_{n}$ in $C$, with $\bar{F}_{j} \leq \bar{F}_{j}$ for $1 \leq j \leq n$, such that $\sharp x \& \geq G=$ $\left(\cap\left\{\theta^{-1}\left(\xi_{j}\right) \times \theta\left(\&_{j}\right): j=1, \ldots, n\right\}\right) \cap\langle\theta\rangle$. If $\nVdash$ is an ultrafilter finer than $\sharp$, then either $\langle\theta\rangle \subseteq K \times K$, contrary to our assumption that $\sharp$ is free, or else there is an index $j, 1 \leq j \leq n$, such that $\theta^{-1}\left(\mathcal{F}_{j}\right) \times \theta\left(\&_{j}\right) \subseteq K \times K$. In the latter case we see easily that $\&_{j} \leqslant 3_{j}$, and $\bar{F}_{j}<\&_{j}$; thus by $(O C)_{1}$ and $(O C)_{2}$, $m_{j}=\left(z_{j} \cap \&_{j}\right)^{\wedge} \in C$. 
Without loss of generality, let $\{1, \ldots, m\}(m \leq n)$ be the indices for which, if $j \in\{1, \ldots, m\}$, there is an ultrafilter $K$ finer than $\sharp$ such that $\theta^{-1}\left(\boldsymbol{F}_{j}\right) \times \theta\left(\&_{j}\right) \subseteq K \times K$. For each $j, 1 \leq j \leq m$, let $m_{j}=\left(\mathfrak{z}_{j} \cap \&_{j}\right)^{\wedge}$; as in the preceding paragraph, $m_{j} \in C$. We next show that $\left.m=n_{\left\{m_{j}\right.}: j=1, \ldots, m\right\} \in C$. Indeed, suppose $j, k \in\{1, \ldots, m\}$. Then there are ultrafilters $k_{j}$ and $k_{k}$ finer than $\&$ such that $m_{j} \subseteq K_{j}$ and $m_{k} \subseteq k_{k}$. Since $K_{j} \times K_{k} \supseteq G$, it follows that either $\left(k_{j} \times k_{k}\right) U<\theta>$ exists, or else $\left(k_{j} \times k_{k}\right) U\left[\theta^{-1}\left(3_{l}\right) \times \theta\left(\& \&_{l}\right)\right]$ exists for some $l \in\{1, \ldots, n\}$, where $\xi_{l} \leqslant \&_{\ell}$. Either alternative leads to the conclusion that $m_{j}<m_{k}$. Applying the same reasoning with indices reversed leads to conclusion $m_{j}<m_{k}$, and by $(O C)_{2}, m_{j} \cap m_{k} \in c$. Since $j$ and $k$ are arbitrary indices in $\{1, \ldots, m\}, m \in C$. Since $m \subseteq \&$, we conclude that $H \in C$ when $\forall$ is a free filter in $\sigma_{\theta, C}$.

If $\sharp x \notin \in \sigma_{O, C}$ is not free, then by $\left.(O C)_{3}\right) \&$ is necessarily of the form $z=A_{1} \cap \dot{x}$, where $\sharp_{1}$ is free and $x \in X$. By our previous results, $\sharp_{1} \in C$. Also, $G \subseteq \sharp x \neq$, where $G$ is the filter described earlier in the proof. Thus $a \subseteq \dot{x} \times H_{1}$ implies $<\theta>U\left(\dot{x} \times \sharp_{1}\right)$ exists or $\left(\theta^{-1}\left(z_{j}\right) \times \theta\left(\&{ }_{j}\right)\right) \cup\left(\dot{x} \times H_{1}\right)$ exists for some $j, 1 \leq j \leq n$. Either way, it follows that $\dot{x} \leq \dot{H}_{1}$. Starting with $a \subseteq H_{1} \times \dot{x}$ leads to the conclusion that $\sharp_{1}<\dot{x}$. Using $(O C)_{2}$ again, we conclude that $\sharp=H_{1} \cap \dot{x} \in C$, and the proof is complete.

The three conditions which characterize ordered Cauchy spaces are all quite natural. The properties "locally convex" and " $\mathrm{T}_{2}$-ordered" are commonly assumed in the study of ordered topological and convergence spaces, and the condition $(O C)_{2}$ is a natural extension to ordered spaces of axiom $\left(C_{3}\right)$ of Definition 1.2. Cauchy structures intrinsic to distributive lattices and lattice ordered groups studied by Ball ([1], [2]) are examples of ordered convergence structures.

Since Cauchy structures are primarily used as a means for constructing completions and compactifications, it is natural to turn our attention now to completions of ordered Cauchy spaces. While it is well known that all $\mathrm{T}_{2}$ Cauchy spaces have $\mathrm{T}_{2}$ completions (indeed, a variety of different completions are described in the literature), it turns out that not all ordered Cauchy spaces have ordered Cauchy completions. In the remainder of this paper, we characterize those ordered Cauchy spaces which do have such completions, and examine several special cases.

\section{ORDERED CAUCHY COMPLETIONS.}

A triple $(\mathrm{X}, \theta, \mathrm{C})$ will be called a poset with $\mathrm{T}_{2}$-ordered Cauchy structure if $(X, \theta)$ is a poset and $C$ a $T_{2}$-ordered Cauchy structure on $(X, \theta)$. Thus a poset with $\mathrm{T}_{2}$ ordered Cauchy structure is required to satisfy condition (OC) ${ }_{3}$ but not $(O C)_{1}$ or $(O C)_{2}$. An increasing, Cauchy-continuous function from one such space to another will be called a morphism. The category having posets with $\mathrm{T}_{2}$-ordered Cauchy structures as objects and morphisms as maps will be designated PCS. Let ocS be the full subcategory of PCS whose objects are the ordered Cauchy spaces.

If $(X, O, C) \in P C S$, let $\theta C$ be the Cauchy structure compatible with the uniform convergence structure $\mu_{\theta, C}=\sigma_{\theta, C} \vee \sigma_{\theta, C}{ }^{-1}$. It follows from Proposition 2.7 that $(\mathrm{X}, \theta, \theta \mathrm{C})$ is $\mathrm{T}_{2}$-ordered. Furthermore, $\mu_{\theta, C}=\mu_{\theta,}, \mathrm{C}$, and so by Proposition 2.6, (X, $\theta, \theta \mathrm{C}$ is an o.c.s. We shall call $\theta \mathrm{C}$ The ordered 
Cauchy modification of $C$ relative to $(x, \theta)$. If $f:(x, \theta, C) \rightarrow\left(x_{1}, \theta_{1}, C_{1}\right)$ is a map in PCS, then it is a simple matter to verify that $f:(x, \theta, \theta C) \rightarrow\left(x_{1}, \theta_{1}, \theta_{1} C_{1}\right)$ is also a morphism.

PROPOSITION 3.1. OCS is a reflective subcategory of PCS relative to the reflector F: PCS $\rightarrow$ OCS defined for objects by $F(X, \theta, C)=(X, \theta, \theta C)$ and fixed on maps.

If an object $(X, \theta, C)$ in PCS is complete, then we shall refer to $C$ as a convergence structure on $X$ and $(X, C)$ as a convergence space. For complete objects in OCS, the $\mathrm{T}_{2}$-ordered property means simply that $O$ is closed in the product convergence on $\mathrm{X} \times \mathrm{X}$. A complete o.c.s. will be called an ordered convergence space; the latter term has the same meaning as " $\mathrm{T}_{2}$-ordered convergence ordered space" in [8]. Let OCON be the full subcategory of ocs whose objects are ordered convergence spaces.

DEFINITION 3.2. An ordered completion $\left(\left(\mathrm{X}_{1}, \theta_{1}, \mathrm{C}_{1}\right), \varphi\right)$ of an o.c.s. $(\mathrm{X}, \theta, \mathrm{C})$ is a complete o.c.s. $\left(\mathrm{X}_{1}, \theta_{1}, \mathrm{C}_{1}\right)$ and a morphism $\varphi:(\mathrm{X}, \theta, \mathrm{C}) \rightarrow$ $\rightarrow\left(x_{1}, O_{1}, C_{1}\right)$ which is an ordered Cauchy embedding (meaning that $\varphi$ and $\varphi^{-1}$ are one-to-one morphisms and $\varphi(X)$ is dense in $X_{1}$ ).

If $\left.\left(x_{1}, \theta_{1}, C_{1}\right), \varphi\right)$ is an ordered completion of $(X, \theta, C)$, then $\left(\left(x_{1}, C_{1}\right), \varphi\right)$ is obviously a Cauchy completion of $(X, C)$, and so it will be convenient to review some aspects of Cauchy completions. Starting with a $\mathrm{T}_{2}$ Cauchy space $(\mathrm{X}, \mathrm{C})$, two filters $\bar{F}, \&$ in $C$ are equivalent if $\cap \& \in C$. Let $X^{*}=\left\{[\xi]: F^{*} \in C\right\}$ be the set of all Cauchy equivalence classes; let $j: X^{*} X^{*}$ be the natural injection, defined by $j(x)=[\dot{x}]$. The completion $\left(\left(x_{1}, c_{1}\right), \varphi\right)$ is in standard form if $X_{1}=X^{*}$ and $\varphi=j$. Reed [10] showed that every Cauchy completion is equivalent (in the usual sense) to one in standard form. The same is, of course, true for ordered completions.

The main goal of this section is to determine which objects in ocs have ordered completions; the full subcategory of ocs determined by these will be denoted by cocs. It is clear that $(X, \theta, C) \in \operatorname{cocs}$ iff $(X, \theta, C)$ is isomorphic to a Cauchy subspace of an object in OCON; we seek, however, an internal characterization of such objects. For this purpose, it is necessary to introduce a new order relation on the Cauchy filters of an o.c.s.

Let $(\mathrm{X}, \theta, \mathrm{C})$ be an o.c.s. For $z \in \mathrm{C}$, let $\mathrm{L}_{\bar{z}}=\{\mathrm{x} \in \mathrm{X}: \dot{\mathrm{x}} \leqslant\}$ and $U_{F}=\{x \in x: F \leq \dot{x}\}$. Note that if $F \cap \dot{y} \in C$, then $L_{F}=Q^{-1}(y)$ and $U_{F}=O(y)$. Now, for $\exists, \& \in C$, define $\exists \in \&$ iff any one of the following is true: $3 \leqslant \&$, or $3 v<L_{\&}>$ exists, or $\& v<U{ }>$ exists. Let $Q$ be the smallest transitive subset of $\mathrm{Cx} C$ containing $\epsilon$. In other words, 3 \& iff there are

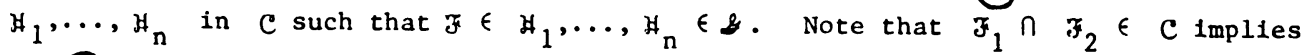
$F_{1} @ 3_{2}$ and $z_{2} \leq z_{1}$; also, if $z<\&$ then $z_{1} Q \&_{1}$ for every $z_{1} \in[\xi]$ and $\xi_{1} \in[\&]$. Before proceeding further, we give an example to illustrate the difference between the relations $\leqslant$ and (

EXANPLE 3.3. Let $\mathrm{X}=\mathrm{R}^{2}-\{(0,0)\}$ be the Euclidean plane with the usual order and with the origin deleted. Let $\mathcal{v}(x)$ be the neighborhood filter at $x$ with respect to the usual topology. Let \& be the filter on $X$ generated by all sets of the form $G_{n}=\left\{(x, 0): 0<x<\frac{1}{n}\right\}$ for $n \in N$, and let $\&$ be generated by all 
sets of the form $H_{n}=\left\{(0, y): 0<y<\frac{1}{n}\right\}$. Let $C$ be the smallest Cauchy structure on $x$ which contains $\mathfrak{v}(x)$ for each $x$ in $x$ along with \& and $\forall$. It is easy to verify that $(X, \theta, C)$ is an o.c.s.; also note that $\& \forall$ and $甘 \xi \&$. However ' ${ }^{\prime} z$ and $U_{H}$ both consist of the closed first quadrant (excluding, of course, the origin), and consequently \& $\bigcirc$ and $\sharp \bigodot^{\&}$.

We now introduce three conditions which are analogous to $(O C)_{1},(O C)_{2}$, and $(O C)_{3}$; we shall show that an o.c.s. $(X, \theta, C)$ has an ordered completion if $f$ it satisfies these new conditions.

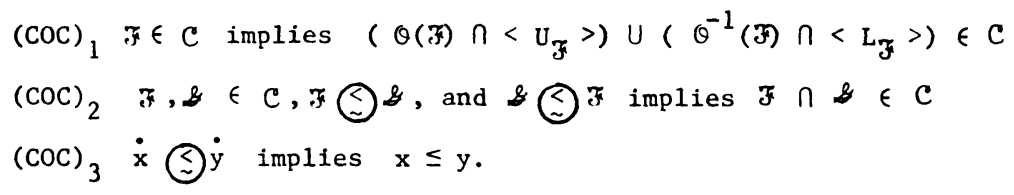

Let $(\mathrm{X}, \theta, \mathrm{C})$ be an o.c.s., and define the preorder $\theta *$ on $\mathrm{X}^{*}$ as follows: $\theta^{*}=\left\{([\because],[\&]): \exists(\&\}\right.$. If $(x, \theta, C)$ satisfies $(\operatorname{COC})_{2}$, then $\theta *$ is a partial order on $X^{*}$; if, in addition, $(\operatorname{COC})_{3}$ is satisfied, then $j:(X, \theta)$ $\rightarrow\left(X^{\star}, \theta^{\star}\right)$ is an order-embedding.

PROPOSITION 3.4. If $\left.\left(X^{*}, \theta_{1}, C_{1}\right), j\right)$ is a completion of $(X, \theta, C)$ in standard form, then $\theta^{*} \subseteq \theta_{1}$.

PRoOF. First, let $\tilde{\theta}=\left\{([\xi],[\&]) \in X^{*} \times X^{*}: \widetilde{F} \leqslant\right\}$. If $([\xi],[\&]) \in \sigma$, then $z \leqslant \&$, and there are $z_{1}, \ldots, z_{n}$ in $C$ such that $\theta(\xi) \cup z_{1}, \ldots, \theta\left(z_{n}\right) \cup \&$ all exist. Thus $3 \times \sharp_{1}, \ldots, \sharp_{n} \times \&$ all have traces on $\theta$. Furthermore, $j(\xi), j\left(H_{1}\right), \ldots, j(\&)$ are all in $c_{1}$, and consequently ([3], $\left.\left[H_{1}\right]\right), \ldots,\left(\left[H_{n}\right],[\&]\right)$ are all in $\theta_{1}$. By transitivity, $([z],[\&]) \in \theta_{1}$, and so $\tilde{\theta} \subseteq \theta_{1}$.

The proof is concluded by observing that if $U\left\langle\mathrm{~L}_{\xi}\right\rangle$ or $\left.\& U<\mathrm{U}_{3}\right\rangle$ exists, then $([\xi],[\&])$ is in the closure of $\tilde{\theta}$. Since $\theta_{1}$ is both closed and transitive, $([\xi],[z]) \in \theta^{*}$ implies $([z],[\xi]) \in \theta_{1}$.

PROPOSITION 3.5. If $(X, \theta, C)$ has an ordered Cauchy completion, then $(X, \theta, C)$ satisfies $(\mathrm{COC})_{1},(\mathrm{COC})_{2}$, and $(\mathrm{COC})_{3}$.

PROOF. Assume that $(X, \theta, C)$ has an ordered Cauchy completion $\left(\left(X^{*}, \theta_{1}, C_{1}\right), j\right)$.

$(\mathrm{COC})_{2}$ If $3, \& \in C, \mathcal{F}^{\&}$, and $\& \mathcal{C}^{3}$, then it follows by Proposition 3.4 that $([\xi],[\&]) \in Q_{1}$ and $([\&],[\xi]) \in \theta_{1}$; since $\theta_{1}$ is a

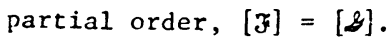

$(\mathrm{COC})_{3}$ If $\dot{x} \odot \dot{y}$, then $\left.(\dot{x}],[\dot{y}]\right) \in \theta_{1}$, and so $(x, y) \in \theta$.

$(\mathrm{COC})_{1}$ By Proposition 3.4, $\theta_{1}(j(\xi) \cap[\dot{\xi}]) \subseteq \theta^{*}(j(\xi) \cap[\dot{\xi}])$. A direct argument shows that $j^{-1}\left(\theta^{*}(j(\xi) \cap[\dot{\xi}])\right)=\theta(\xi) \cap\left\langle\mathbb{H}^{\prime}\right\rangle$. But $\theta_{1}(j(\xi) \cap[\dot{\xi}]) \cup \theta_{1}^{-1}(j(\xi) \cap[\dot{\xi}]) \in C_{1}$, and consequently $\left(\theta(F) \cap\left\langle U_{F^{F}}>\right) U\left(\theta^{-1}(\xi) \cap\left\langle L_{z}\right\rangle \in C\right.\right.$. I

It was shown that the o.c.s. $(X, \theta, C)$ of Example 3.3 fails to satisfy $(\operatorname{COC})_{2}$, and thus we see that $\operatorname{COCS}$ is a proper subcategory of ocs. 
We next show that the conditions $(\mathrm{COC})_{1},(\mathrm{COC})_{2}$, and $(\mathrm{COC})_{3}$ are also sufficient for the existence of an ordered completion. First, recall Wyler's completion $\left(\left(X^{*}, C_{W}\right), j\right)$ of an arbitrary $T_{2}$ Cauchy space $(X, C)$, defined by $C_{W}=$ $\{j(3) \cap[\dot{\xi}]: x \in C\}$.

THEOREM 3.6. Let $(\mathrm{X}, \theta, \mathrm{C})$ satisfy $(\mathrm{COC})_{1},(\mathrm{COC})_{2}$, and $(\mathrm{COC})_{3}$. Then $\left(X^{*}, Q^{*},\left(* C_{W}\right)\right.$ is an ordered completion of $(X, \theta, C)$. If $f:(X, \theta, C) \rightarrow$ $\left(x_{1}, \theta_{1}, C_{1}\right)$ is a morphism and $\left(x_{1}, \theta_{1}, C_{1}\right) \in O C O N$, then there is a unique morphism $f^{*}:\left(X^{*}, \theta *, \theta * C_{W}\right) \rightarrow\left(X_{1}, \theta_{1}, C_{1}\right)$ such that $f=f^{*} \circ j$.

PROOF. We have previously observed that conditions $(\mathrm{COC})_{2}$ and $(\mathrm{COC})_{3}$ guarantee that $\mathrm{j}:(\mathrm{X}, \theta) \rightarrow\left(\mathrm{X}^{*}, \theta^{*}\right)$ is an order embedding. It is also clear that $j:(X, C) \rightarrow(X *, \theta * C W)$ is Cauchy-continuous, and that $j(X)$ is dense in $X *$. The fact that $j^{-1}$ is also Cauchy-continuous follows from (COC) 1 and the result (used also in the preceding proof) that $\theta(\bar{F}) \cap\left\langle U_{\mathcal{F}}\right\rangle=j^{-1}(\theta *(j(\mathfrak{F}) \cap[\dot{\mathcal{F}}]))$ (along with the dual equality).

Given a function $f$ as specified, let $f *$ be the natural extension defined by $f *([\xi])=y_{z}$, where $f(\xi) \cap \dot{y}_{3} \epsilon C_{1}$. Then $f *:\left(X *, C_{w}\right) \rightarrow\left(X_{1}, C_{1}\right)$ is Cauchy-continuous by the well-known extension properties of Wyler's completion (see $[5])$, and $f *:\left(X^{*}, \theta * C_{w}\right) \rightarrow\left(X_{1}, C_{1}\right)$ is Cauchy-continuous by Proposition 3.1 . To show that $f *$ is increasing, we first note that $\& \&$ in $C$ implies $f(\xi) @ f(\xi)$ in $c_{1}$. Since $c_{1}$ is complete, we have also that $f(\xi) \lesssim f(\xi)$, and it follows from Proposition 1.2, [8] that. $f *([3])=y_{f(3)} \leq y_{f(\&)}=f *([\&])$.

Henceforth, the ordered completion of $(X, \theta, C)$ defined in Theorem 3.6 will be denoted by $\left(X^{*}, \theta^{*}, C^{*}\right)$ rather than $\left(X^{*}, \theta^{*}, \theta^{*} C_{w}\right)$.

Following [4], we define an ordered completion $\left(\left(X^{*}, \theta_{1}, C_{1}\right), j\right)$ to be order-strict if, for any ordered completion $\left(\left(X^{*}, \theta_{2}, C_{2}\right), j\right)$ in standard form, $\theta_{1} \subseteq G_{2}$. From Proposition 3.4, it follows that the ordered completion of Theorem 3.6 is order strict.

COROLLARY 3.7. For an o.c.s. (X, $\theta, C)$, the following statements are equivalent.

(1) $(\mathrm{X}, \theta, \mathrm{C}) \in \operatorname{cocs}$

(2) $(\mathrm{X}, \mathrm{C}, \mathrm{C})$ satisfies conditions $(\mathrm{COC})_{1},(\mathrm{COC})_{2}$, and $(\mathrm{COC})_{3}$.

(3) $(X, \theta, C)$ has an order-strict ordered completion.

(4) $\left(X^{*}, O^{*}, C^{*}\right)$ is an ordered completion of $(X, \theta, C)$.

Without repeating the relevant definitions here, we remark that, in the terminology of [5], the functor $\mathrm{K}$ : $\operatorname{cocs} \rightarrow \operatorname{OCON}$, defined by $\mathrm{K}(\mathrm{X}, \theta, \mathrm{C})=$ $\left(X^{*}, \theta^{*}, C^{*}\right)$ is an order-strict completion functor, and consequently that COCS is a completion subcategory of OCS. We shall henceforth refer to $\left(\left(X^{*}, Q^{*}, C^{*}\right), j\right)$ as the fine ordered completion of $(X, \theta, C)$, and $K$ will be called the fine ordered completion functor.

4. UNIFORMIZABLE ORDERED CAUCHY SPACES.

Let $(x, \theta, U)$ be a poset $(x, \theta)$ equipped with a $T_{2}$ uniformity $U$. Then $(x, \theta, U)$ is a uniform ordered space (Nachbin, [9]) if there is a quasiuniformity $\delta$ on $x$ such that $u=\delta \cup g^{-1}$ and $\theta=n \delta$; such a quasiuniformity is said to determine $(x, \theta, \mathcal{U})$. The set of $U$-Cauchy filters is denoted by $\mathcal{U}$. 
PROPOSITION 4.1. Let $(X, \theta, U)$ be a uniform ordered space determined by a quasiuniformity $\delta$. Then the following conditions hold.

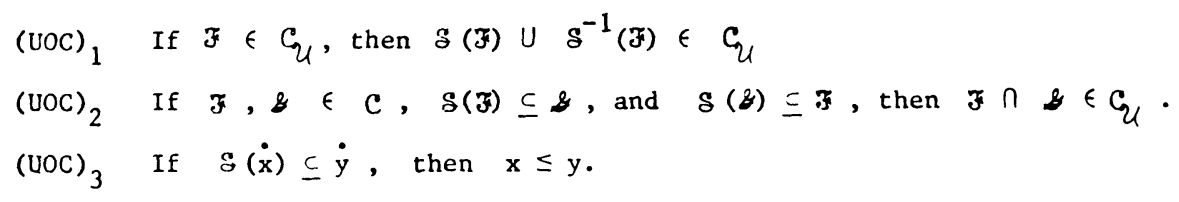

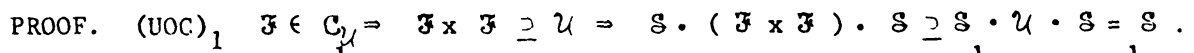

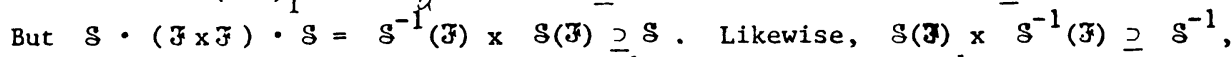

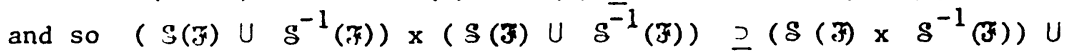
$\left(g^{-1}(\xi) \times g(\xi)\right) \geq \& U g^{-1}=u$. $(\mathrm{UOC})_{2} \xi \times \exists \geq g$ and \& \& $\& \delta \Rightarrow(\xi \times 3) \cdot \delta \cdot(\& \times \&)=$

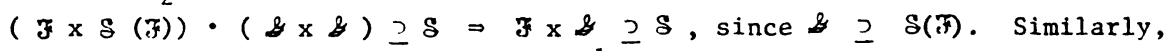

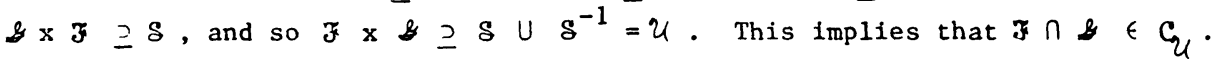
(UOC) $_{3} \quad g(\dot{x}) \subseteq \dot{y} \Rightarrow \dot{x} x \dot{y} \geq S \Rightarrow(x, y) \in \cap \quad S \Rightarrow x \leq y$.

THEOREM 4.2. If $(X, \theta, U)$ is a uniform ordered space, then $\left(X, \theta, C_{U}\right)$ is an o.c.s.

PROOF. Let $\&$ be a quasiuniformity on $x$ that determines $(X, \theta, U)$. Let $\exists<\xi$ in $C_{\mathcal{U}}$. Then, by Proposition 2.5, $₹ \times \& \in \sigma_{\theta, C_{U}}$. Clearly $\&$ generates a quasiuniform convergence structure coarser than $\sigma \theta, c_{\mathcal{U}}$, and therefore $\exists \times \& \geq S$. Thus $\delta(\exists) \subseteq \&$. Conditions (UOC) ${ }_{2}$ and ${ }^{-}(U O C)_{3}$ thus imply conditions $(O C)_{2}$ and $(O C)_{3}$, respectively. But $(O C)_{1}$ follows from (UNC) 1 and the fact that $S \subseteq\langle\theta\rangle$. Thus $\left(X, \theta, C_{U}\right)$ is an o.c.s. by Theorem 2.9.

An o.c.s. (X, $\theta, C)$ is uniformizable if there is a $T_{2}$ uniform ordered space $(x, O, U)$ such that $c=\boldsymbol{C}_{U}$.

THEOREM 4.3. Each uniformizable o.c.s. has an ordered completion.

PROOF. Let $(X, \theta, C)$ be a uniformizable o.c.s., and let $(X, \theta, U)$ be a compatible uniform ordered space; let $\delta$ be a quasi-uniformity which determines $(x, \vartheta, \mathcal{U})$. Let $z \in C$ and $x \in U_{F}$. Then $z \leqslant \dot{x}$, and it follows from the preceding proof that $\delta(\mathcal{F}) \subseteq \dot{x}$, and consequently $S(\mathcal{F}) \subseteq\left\langle U_{\mathcal{F}}\right\rangle$. Also

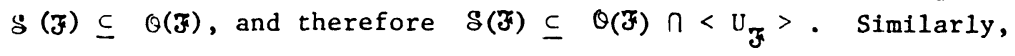
$S^{-1}(\xi) \subset \theta^{-1}(\Im) \cap\left\langle L_{\zeta}\right\rangle$. Thus (COC) 1 follows from (UOC) 1 . A straightforward argument shows that $\delta(\bar{F}) \subseteq \&$ whenever $\bar{F} \&$, and so $(\mathrm{COC})_{2}$ and $(\mathrm{COC})_{3}$ follow from $(\mathrm{UOC})_{2}$ and $(\mathrm{UOC})_{3}$, respectively. The conclusion follows by Theorem 3.6 .

It is natural to ask whether the fine completion functor $K$, when restricted to uniformizable o.c.s.'s, preserves uniformizability. As the next example shows, the answer is no. The same example shows that the fine completion preserves neither regularity nor total boundedness.

EXAMPLE 4.4. Let $E$ be the Euclidean plane with the usual topology and partial order: $Q_{E}=\{(a, b),(c, d)): a=c$ and $\left.b \leq d\right\}$. Note that the elements in $E$ are $Q_{E}$-related iff they lie on the same vertical line. Let $Y=U\left\{L_{n}: n \in N\right\} U$ 
$\{(0,0)\}$, where $L_{n}=\left\{\left(\frac{1}{n}, y\right): 0 \leq y \leq \frac{1}{n}\right\}$; let $\left(Y, \theta^{\prime}, \theta^{\prime}\right)$ be the compact, $T_{2}-$ ordered topological space (considered as an object in OCON) with order and topology inherited from E. Let $S=\left\{\left(\frac{1}{n}, \frac{1}{n}\right): n \in N\right\}$ and let $X=Y-S$. The order and Cauchy structures which $X$ inherits from $Y$ are denoted by $\theta$ and $\theta$, respectively. Since $\left(Y, \theta^{\prime}, Q^{\prime}\right)$ is a compact, $T_{2}$-ordered completion of $(X, O, \theta)$, $(X, \Theta, B)$ is a uniformizable o.c.s.

Now consider the fine completion $\left(\left(X^{*}, \theta^{*}, \theta^{*}\right), j\right)$ of $(X, \theta, \theta)$. There is an obvious correspondence between the sets $X *$ and $Y$ relative to which the equivalence classes of non-convergent filters in $X^{*}$ correspond to the subset $S$ of $Y$. Let \& be the Frechet filter on $Y$ of the sequence $\left(\frac{1}{n}, \frac{1}{n}\right)$, and let $G$ be the corresponding filter on $X^{*}$. Although \& converges to $(0,0)$ in $\left(Y, Q^{\prime}\right)$, $a$ is non-convergent relative to $\left(X^{*}, D^{*}\right)$. If $p \in X^{*}$ is the equivalence class of filters converging in $(X, D)$ to the origin, then the closure of the p-neighborhood filter in $\left(X^{*}, D *\right)$ is nonconvergent, and so $\left(X^{*}, D^{*}\right)$ is not regular, and consequently not uniformizable.

From the fact that $\left(Y, \theta^{\prime}, \theta^{\prime}\right)$ is compact, it also follows that $(X, \theta, \theta)$ is totally bounded (meaning that every ultrafilter on $X$ is in $\theta$ ). Since no ultrafilter finer than $G$ converges in $\left(X^{*}, \Delta^{*}\right)$, we see that total boundedness is not preserved by the fine completion functor.

Although no definition of "regularity" has been given for ordered Cauchy spaces, the space $(X, \theta, \theta)$ as an ordered Cauchy subspace of a $\mathrm{T}_{2}$-ordered, compact topological space, would be "regular" by any reasonable definition of that term. Since $\left(X^{*}, G^{*}, D^{*}\right)$ is not regular (in the usual convergence space sense) no reasonable notion of regularity is preserved by the fine completion functor.

\section{TOTALLY ORDERED CAUCHY SPACES.}

We begin with a simple condition which is sufficient for the existence of an ordered completion of an o.c.s.

DEFINITION 5.1. An o.c.s. (X, $Q, C)$ satisfies Condition (A) if, whenever

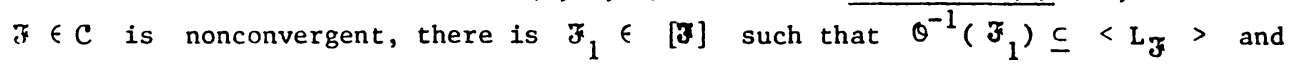
$O\left(\mathrm{~J}_{1}\right) \subseteq\left\langle U_{3}>\right.$.

PROPOSITION 5.2. If $(X, \theta, C)$ is an o.c.s. which satisfies Condition (A), then:

(1) The relations $($ and $\lesssim$ on C colncide.

(2) $(\mathrm{COC})_{1} \oplus(\mathrm{OC})_{1}$

(3) $(\mathrm{COC})_{2} \oplus(\mathrm{OC})_{2}$

(4) $(\mathrm{COC})_{3} \bullet(\mathrm{OC})_{3}$

(5) $(\mathrm{X}, \theta, C)$ has an ordered completion.

PROOF. (1) Suppose $J U<\mathrm{L}_{\&}>$ exists. If \& converges to $y$, then $L_{\&}=\theta^{-1}(y)$, and $3<\&$ follows immediately. If $\&$ is nonconvergent, Condition (A) guarantees that $3 \cup Q^{-1}(\&)$ exists, and so $3<\&$. Similar reasoning shows that $z<\&$ whenever $\& U<U_{\exists}>$ exists. Thus it is clear that F $\&$ implies $\& \sim$. The converse is always true. Statements (2), (3), (4), and (5) are easy corollaries of (1). 
Note that if $(X, \theta, C)$ is an o.c.s. satisfying Condition (A), then $\theta *=\left\{\left(\left[z^{*}\right],[\&]\right) \in X^{*} \times X^{*}: F \leqslant \&\right\}$.

The term totally ordered Cauchy space (abbreviated t.o.c.s.) will be used for any o.c.s. (X, $\theta, C)$ for which $\theta$ is a total order.

PROPOSITION 5.3. If 3 and $\&$ are filters on a totally ordered set $(\mathrm{X}, \theta)$, then either $\theta(\xi)$ IJ \& exists, or $\theta^{-1}$ (अ) $U \&$ exists.

PROOF. If both fail to exist, there is $F \in F$ and $G \in \&$ such that

$\theta(F) \cap G=\phi$ and $\theta^{-1}(F) \cap G=\phi$, which means that $\left(\theta(F) \cup \theta^{-1}(F)\right) \cap G=\phi$. Since $F$ is nonempty, $\theta(F) \cup Q^{-1}(F)=X$, and $X \cap G=\phi$ is impossible.

COROLlaRY 5.4. Let $(\mathrm{X}, \theta, \mathrm{C})$ be a t.o.c.s. and $F, \& \in \mathrm{C}$. If $F<z$, then either $\theta(\xi) \cup \&$ exists, or else $z \in[\&]$.

PROOF. If $G(F) \cup \&$ fails to exist, then $Q(\&) \cup \mathbb{F}$ exists, and so $\&<\xi$. But $z<\hat{\&}$ is assumed, so by $(\mathrm{OC})_{2}, \xi \in[\&]$.

PROPOSITION 5.5. A t.o.c.s. satisfies Condition (A).

PROOF. Let $(X, \theta, C)$ be a t.o.c.s., and let $\in C$ be nonconvergent. If

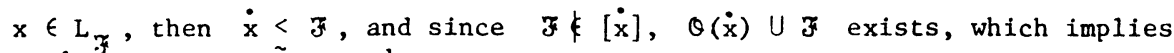
$G^{-1}(\vec{F}) \subseteq \dot{\mathrm{x}}$. Thus $\hat{\theta}^{-1}(\bar{J}) \subseteq\left\langle\mathrm{L}_{\overrightarrow{3}}\right\rangle$. This, along with the dual argument, proves Condition (A).

COROLlARY 5.6. Every t.o.c.s. (X, O, C) has an ordered completion, and every ordered completion of $(X, \theta, C)$ is totally ordered.

PROOF. The existence of an ordered completion follows immediately from Proposition 5.2 and 5.5. The completion ( $\left.X^{*}, \theta^{*}, C^{*}\right)$ is totally ordered by Proposition 5.3 and the remark following Proposition 5.2. Since the total order $Q^{*}$ is the smallest allowable order for an ordered completion in standard form, it is the only possible order for an ordered completion in standard form.

\section{AN ORDERED CONPACTIFICATION.}

Every $\mathrm{T}_{2}$-ordered compactification of an ordered topological or convergence space can be regarded as the completion of a certain totally bounded o.c.s., and so the entire subject of $\mathrm{T}_{2}$-ordered compactifications lies within the scope of our present investigation. Our immediate goal, however, is rather modest; we shall formulate the ordered convergence compactification constructed in [8] as an ordered Cauchy space completion, thereby gaining some further insight into its properties.

Let $(X, \theta, \rightarrow)$ be an ordered convergence space (i.e., an object in ocoN); the notation " $\rightarrow x^{\prime \prime}$ indicates that converges to $x$ in this space. Let $C^{\rightarrow}$ be the complete Cauchy structure on $x$ consisting of all convergent filters relative to $\rightarrow$, and (following [8]) let $X^{\prime}$ be the set of all nonconvergent, maximal convex filters on $(X, \theta, \rightarrow)$. In [8], an ordered convergence space $(\mathrm{X}, \theta, \rightarrow)$ is defined to be strongly $\mathrm{T}_{2}$-ordered, if the following property and its dual are satisfied: If $\rightarrow \mathrm{x}, \& \in \mathrm{x}^{\prime}$ and $\theta(\xi) \cup \&$ exists, $\theta^{-1}(\&) \subseteq \dot{\mathrm{x}}$.

With each ordered convergence space $(X, \theta, \rightarrow)$, we associate the Cauchy structure $\&=\mathrm{C}^{\rightarrow} U\left\{\xi \in \mathrm{F}(\mathrm{X})\right.$ : there is $\& \in \mathrm{X}^{\prime}$ such that $\mathrm{G} \subseteq \mathrm{F}^{\prime}$. Note that $\mathcal{A}$ is the finest Cauchy structure compatible with $\rightarrow$ which is both totally 
bounded and locally convex relative to $\theta$.

A Cauchy completion of $(X, D)$ in standard form is constructed as follows. If $A \subseteq X$, let $\tilde{\Lambda}=j(X) \cup\left\{[F] \in X^{*}: F \in X^{\prime}\right.$ and $A \in \mathcal{F}$. If $\tilde{F} \in F(X)$, let $\tilde{\xi}$ be the filter on $X^{*}$ generated by $\{\tilde{F}: F \in \mathcal{F}\}$. Let $\tilde{\mathbb{Q}}=\left\{G \in F\left(X^{*}\right)\right.$ : there is $z \in \mathbb{D}$ such that $\tilde{z} \leq \mathbb{G}$.

PROPOSITION 6.1. [8] For any ordered convergence space $(\mathrm{X}, \theta, \rightarrow),\left(\mathrm{X}^{\star}, \tilde{\theta}^{2}\right)$ is a $\mathrm{T}_{2}$ compactification of $(\mathrm{X}, \rightarrow)$.

PROPOSITION 6.2. A convergence ordered space $(X, \theta, \rightarrow)$ is strongly $\mathrm{T}_{2}$-ordercd iff $(\mathrm{X}, \theta, Q)$ is an o.c.s. which satisfies Condition (A).

PROOF. If $(X, \theta, \theta)$ is an o.c.s., then $(X, \theta, \rightarrow)$, which is its associated convergence space, is clearly an ordered convergence space. It is also clear that Condition (A) applied to $(X, \theta, D)$ implies the strongly $T_{2}$-ordered property for $(\mathrm{X}, \mathrm{\theta}, \rightarrow)$.

Conversely, assume $z \leqslant$ relative to $(X, \theta, D)$, where $\&$, $\&$ are both convex, and either which is non-convergent is assumed to be maximal convex. A straightforward argument based on the strongly $\mathrm{T}_{2}$-ordered property leads to exactly one of the following conclusions:

(1) There are $x, y \in x$ such that $z \rightarrow x, \& \rightarrow y$, and $\theta(\dot{x}) \subseteq \dot{y}$.

(2) There is $x \in X$ such that $\rightarrow x, \&$ is nonconvergent, and $\theta(\dot{x}) \subseteq \&$.

(3) There is $\mathrm{y} \in \mathrm{X}$ such that $\& \rightarrow \mathrm{y}, \quad$ is nonconvergent, and $\theta(\&) \subseteq \dot{y}$.

(4) Both $\exists$ and $\&$ are nonconvergent, and $\theta(\Im) \subseteq \&$.

With the help of these results, conditions $(O C)_{1},(O C)_{2}$, and $(O C)_{3}$ are easily verified, and so $(X, \theta, \theta)$ is an o.c.s. Finally, if $\exists \in \theta$ is nonconvergent, $F_{1}$ is the maximal convex filter coarser than , and $\dot{x} \leqslant$, then statement (2) above gives the result $\theta(\dot{x}) \subseteq \vec{F}_{1}$, or equivalently $\dot{x} \geq \theta^{-1}(\mathfrak{x})$, which implies $\left\langle\mathrm{L}_{Z^{3}}>\geq Q^{-1}\left(\xi_{1}\right)\right.$. This, along with the dual argument establishes Condition (A).

Combining the preceding results and Proposition 2.8, [8], we obtain:

THEOREM 6.3. If an ordered convergence space $(X, \theta, \rightarrow)$ is strongly $\mathrm{T}_{2}$-ordered, then $\left(\left(\mathrm{X}^{*}, \theta^{*}, \tilde{\otimes}\right), \mathrm{j}\right)$ is an order-strict, $\mathrm{T}_{2}$-ordered compactification of $(X, G, \rightarrow)$.

Under the assumptions of Theorem 6.3, (X, $\theta, \rightarrow)$ also has an extension of the form $\left(X^{*}, \theta^{*}, B^{*}\right)$, which is the fine completion of $(X, \theta, \theta)$. From Theorem 3.6, it follows that $\theta^{*}$ is finer than $\tilde{\theta}$. If $\theta *$ is totally bounded, then $\left(X^{*}, \theta^{*}, Q^{*}\right)$ is also a compactification, and indeed $\theta^{*}=\tilde{\theta}$ in this case.

PROPOSITION 6.4. Under the assumptions of Theorem $\left.6.3,\left(X^{*}, \theta^{*}, \theta^{*}\right), j\right)$ is the largest $\mathrm{T}_{2}$-ordered compactification of $(\mathrm{X}, \theta, \rightarrow)$ iff $\theta^{*}$ is totally bounded. When this happens, $\left.\left(X^{*}, Q^{*}, Q^{*}\right), j\right)$ coincides with the compactification $\left(X^{*}, \theta^{*}, \tilde{Q}\right)$ of $(X, \theta, \rightarrow)$ constructed in [8].

It is shown in [8] that, under the assumptions of Theorem $6.3,\left(X^{*}, \theta_{*}, 0\right)$ Is the largest "relatively regular" $\mathrm{T}_{2}$-ordered compactification of $(\mathrm{X}, \theta, \rightarrow)$. There is not, in general, a largest $\mathrm{T}_{2}$-ordered compactification of a strongly $\mathrm{T}_{2}$-ordered convergence space as the concluding example shows. 
EXAMPLE 6.5. This example is based on Example 4.4, and the same definitions and notation will be used here. Let $(\mathrm{X}, \theta, \rightarrow)$ be the $\mathrm{T}_{2}$-ordered topological space compatible with $(X, \theta, Q)$; in other words, $\rightarrow$ is the relativization to $X$ of the usual topology on $E$. It turns out that $d$ is the finest locally convex totally bounded Cauchy structure on $\mathrm{X}$ compatible with $(\mathrm{X}, \theta, \rightarrow)$. The compactification $\left(X^{*}, Q^{*}, \mathscr{D}^{\prime}\right)$ is equivalent to the compactification $\left(Y, Q^{\prime}, \mathscr{S}^{\prime}\right)$, while the fine completion $\left(X^{*}, \theta^{*}, \theta^{*}\right)$ of $(X, \theta, d)$ is not totally bounded. Thus, in general, $\mathscr{Q} *$ and $\tilde{\mathscr{D}}$ are distinct. There is no largest $\mathrm{T}_{2}$-ordered compactification of $(\mathrm{X}, \theta, \rightarrow)$. Indeed, suppose $\mathrm{C}^{\prime \prime}$ is the complete Cauchy structure on $Y$ which agrees with $C^{\prime}$, except that the filter \& and all finer filters are assigned to converge to $(1,0)$ instead of $(0,0)$. Then (Y, $\left.\theta^{\prime}, C^{\prime \prime}\right)$ is a $T_{2}$-ordered compactification of $(X, \theta, \rightarrow)$ which is neither larger nor smaller than $\left(Y^{\prime}, Q^{\prime}, C^{\prime}\right)$.

By altering the order structure in the preceding example, one can get quite different results. Suppose $\left(X, \theta_{1}, \rightarrow\right)$ is obtained by leaving $X$ and $\rightarrow$ unchanged, but replacing $\theta$ by $\theta_{1}$, the usual order of the Euclidean plane. It turns out that $\left(\mathrm{X}, \theta_{1}, \rightarrow\right)$ is strongly $\mathrm{T}_{2}$-ordered and that $\theta$ is again the finest compatible totally bounded locally convex Cauchy structure. In this case the fine completion $\left(X^{*}, \theta_{1}^{*}, Q^{*}\right)$ of $\left(X, \theta_{1}, \&\right)$ is totally bounded. Thus, by Proposition $6.4,\left(X^{*}, \theta_{1}^{*}, Q^{*}\right)$ coincldes with the compactification constructed in [8], and is the largest $\mathrm{T}_{2}$-ordered compactification of $\left(\mathrm{X}, \theta_{1}, \rightarrow\right)$.

\section{REFERENCES}

1. BALL, R.N. Convergence and Cauchy Structures on Lattice Ordered Groups, Trans. Amer. Math. Soc. 259 (1980), 357-392.

2. BALL, R.N. Cut Completions of Lattice Ordered Groups by Cauchy Constructions, Ordered Groups, M. Dekker, Lecture Notes in Pure and Appl. Math. Vol. 62 (1980), 81-92.

3. Cook, C.H. and FISHER, H.R. Uniform Convergence Structures, Math. Annalen 173 $(1967), 290-306$.

4. FLETCHER, P. and LINDGREN, W. Quasi-uniform Spaces, Lecture Notes in Pure and Appl. Math. Vol. 77 (1982).

5. FRIC, R. and KENT, D.C. Completion Functors for Cauchy Spaces, Internat. J. Math. \& Math. Sci. 4 (1979), 589-604.

6. GäHLER, W. Grundstrukturen der Analysis, Band I, Akademie-Verlag, Berlin (1977).

7. KELLER, H.H. Die Limes - Uniformisierbarkeit der Limesräume, Math. Annalen 176 $(1968), 334-341$

8. KENT, D.C. and RICHARDSON, G.D. A Compactification for Convergence Ordered Spaces, Canadian Math. Bu11. 27 (1984) 505-513.

9. NACHBIN, L. Topology and Order, Van Nostrand Math. Studies, No. 4, Princeton, N. J. (1965).

10. REED, E.E. Completions of Uniform Convergence Spaces, Math. Annalen 194 (1971), 83-108. 


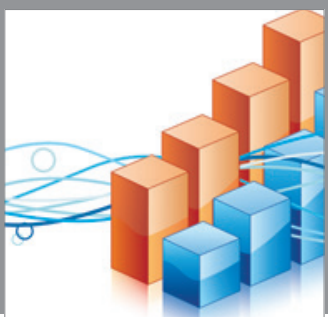

Advances in

Operations Research

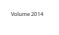

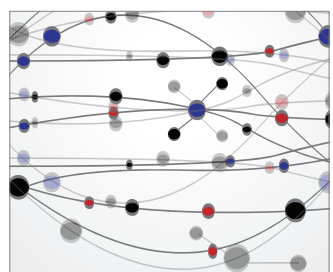

\section{The Scientific} World Journal
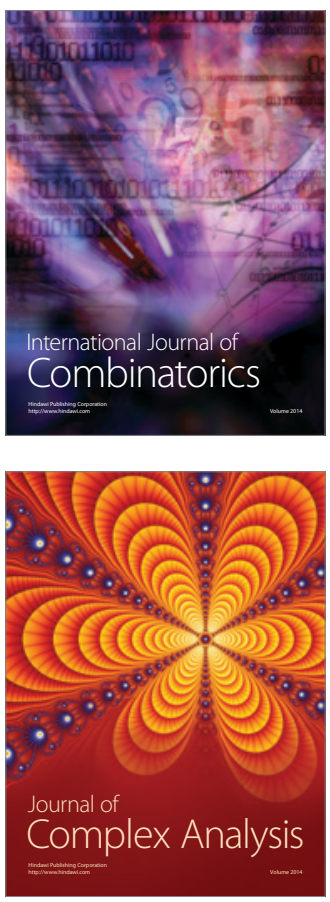

International Journal of

Mathematics and

Mathematical

Sciences
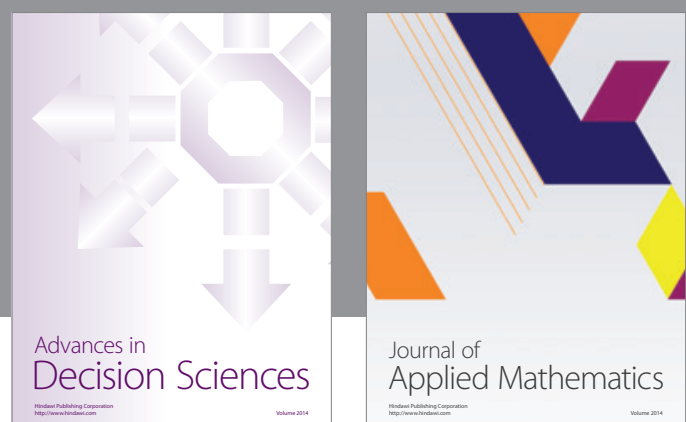

Journal of

Applied Mathematics
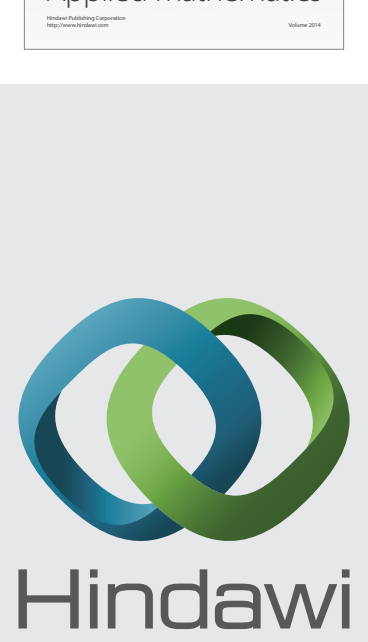

Submit your manuscripts at http://www.hindawi.com
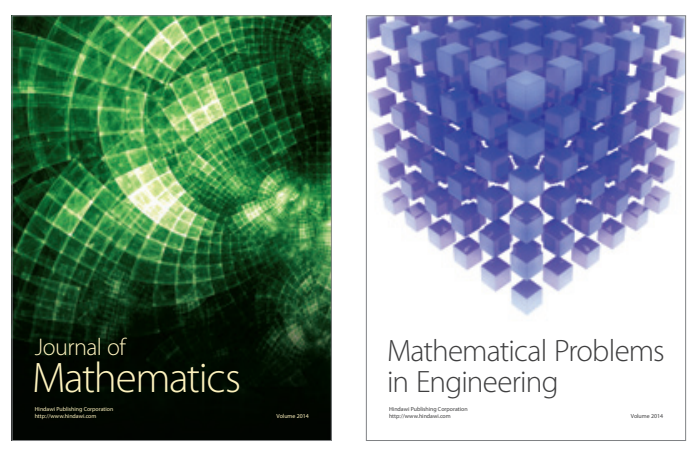

Mathematical Problems in Engineering
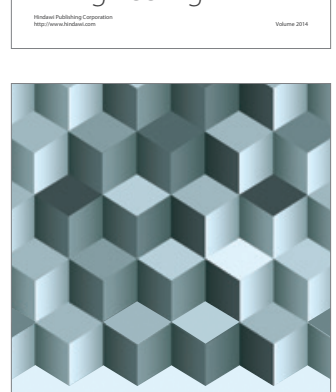

Journal of

Function Spaces
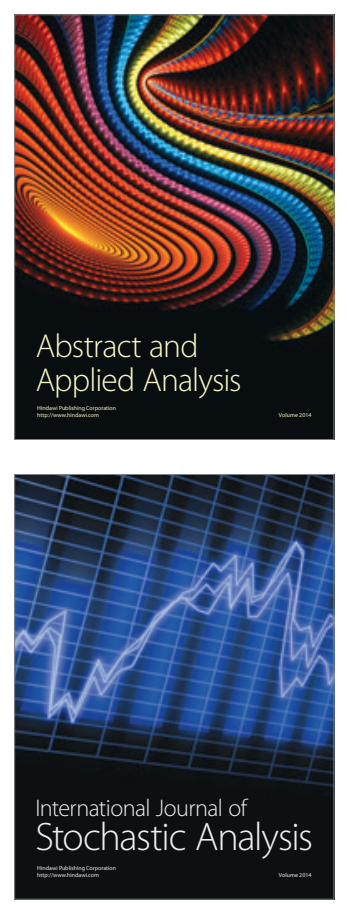

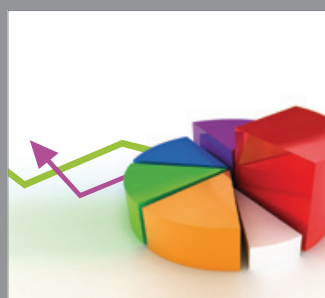

ournal of

Probability and Statistics

Promensencen
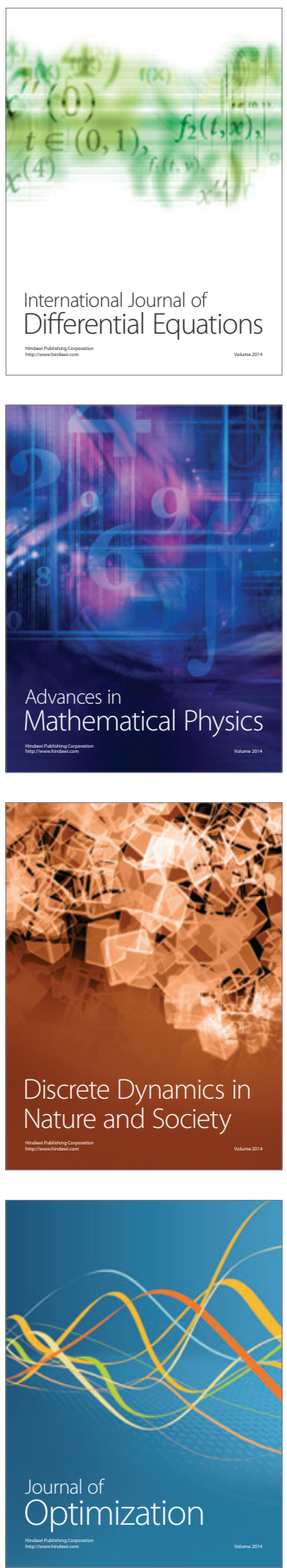